13. Hayashi Y. Ando T. 'Utagawa E. et al. Western blot (immunoblot) assay of small, round-structured virus associated with an acute gastroenteritis outbreak in Tokyo. J Clin Microbiol 1989;27:1728-33.

14. Kurtz JB, Lee TW. Craig JW. Reed SE. Astrovirus infection in volunteers. J Med Virol 1979:3:22I-30.

15. Oishi I. Three-year survey of the epidemiology of rotavirus, enteric adenovirus, and some small spherical viruses, including "Osaka agent" associated with infantile diarrhea. Biken J 1985:28:9-19.

\section{Note Added in Proof}

The astrovirus strain from this outbreak reacted in a serotyping EIA (Noel J, personal communication) with rabbit antisera raised to serotype 6 provided by J. B. Kurtz (Lee TW, Kurtz JB. Prevalence of human astrovirus serotypes in the Oxford region 1976-1992, with evidence of two new serotypes. Epidemiol Infect 1994; $112: 187-93$ ).

\title{
Viral Replication and Development of Specific Immunity in Macaques after Infection with Different Measles Virus Strains
}

\author{
Robert S. van Binnendijk, Roger W. J. van der Heijden, \\ Geert van Amerongen, Fons G. C. M. UytdeHaag, and \\ Albert D. M. E. Osterhaus
}

\author{
Department of Virology. Erasmus Universitv. Rotterdam; Central \\ Animal Laboratory. National Institute of Public Health and \\ Environmental Protection. Bilthoven. Netherlands
}

\begin{abstract}
Cynomolgus monkeys (Macaca fascicularis) were experimentally infected with a wild type measles virus (MV) strain (MV-BIL). Following intratracheal inoculation with different infectious doses, the virus could be isolated from peripheral blood mononuclear cells (PBMC), lung lavage cells, and pharyngeal cells. The kinetics of the cell-associated viremia was similar in all infected animals. They developed specific serum IgM, IgG, and neutralizing antibody responses as well as MV-specific T cell-mediated immunity. Monkeys infected intratracheally or intramuscularly with the wild type MV-Edmonston or the attenuated MV-Schwartz strain showed a lower level of PBMC-associated viremia and less pronounced specific IgM responses. Nine months after infection with MV strains, all of the monkeys were protected from intratracheal reinfection with MV-BIL. This monkey model is suitable for study of new generations of vaccines and vaccination strategies for measles.
\end{abstract}

In humans, measles is believed to confer lifelong immune protection against reexposure to measles virus (MV). This protection appears largely to be based on persistence of $\mathrm{MV}$ neutralizing antibodies and the presence of MV-specific cytotoxic $\mathrm{T}$ lymphocytes (CTL) [1, 2]. Vaccination against measles with live attenuated measles vaccines likewise aims at the induction of lifelong immune protection based on the same immune defense mechanism. However, even in industrialized countries where measles has largely disappeared as a consequence of vaccination with live attenuated measles vaccines, outbreaks of measles continue to occur [3-5]. Of more importance, due to low vaccination coverage and the interference of transplacentally acquired maternal antibodies with replication of vaccine virus, measles remains a major cause of serious disease and mortality in developing countries, annually killing $\sim 1.5$ million children $[6,7]$. Thus, MV continues to circulate in developing countries as well as

Received 24 November 1993; revised 22 March 1994.

Financial support: United Nations Development Programme, World Health Organization (V21/181/35)

Reprints or correspondence: Prof. A. D. M. E. Osterhaus, Dept. of Virology. Erasmus University Rotterdam, P.O.Box 1738, 3000 DR Rotterdam, Netherlands.

The Journal of Infectious Diseases 1994;170:443-8

C 1994 by The University of Chicago. All rights reserved. $0022-1899 / 94 / 7002-0032 \$ 01.00$ in countries with high vaccination coverage rates. As a consequence, the World Health Organization has reevaluated strategies for control and eradication of measles [7].

The present study was aimed toward development of a suitable animal model that would provide more insight into the pathogenesis of measles, functional aspects of humoral and cellular immune responses, and possible molecular correlates of virus attenuation. Apart from humans, several nonhuman primate species can be experimentally infected with MV or can contract measles when exposed to infected humans, thereby spreading the virus from monkey to monkey [8]. Among these, marmosets (Saguinus mystax) are the most susceptible to infection with wild type MV. However, clinical signs and gross pathologic findings that accompany the usually fatal disease in these animals, lack the characteristic features of measles in humans [9]. In a number of other simian species, such as cynomolgus and rhesus monkeys (Macaca fascicularis and Macaca mulatta, respectively), natural and experimental infection with wild type MV causes disease of varying severity but with a pathogenesis similar to that of measles in humans [8]. These species may be suitable candidates in which to study the pathogenesis of wild type and attenuated MV infections and the mechanisms of immune-mediated protection they induce. In this study, we evaluated cynomolgus monkeys for possible use as an animal model for study of MV. 


\section{Materials and Methods}

Wild type MV-BIL isolate. During an outbreak of measles at a high school in Bilthoven, Netherlands, MV was isolated from peripheral blood mononuclear cells (PBMC) and nasopharyngeal washings from patients. MV-BIL was isolated in a continuously growing human B lymphoblastoid cell line (B-LCL) from PBMC of a patient in the outbreak [10]. Viruses isolated in the course of these studies were characterized as MV by negativecontrast electron microscopy and by immunofluorescence staining using monoclonal and rabbit polyclonal anti-MV antisera as described [11, 12]. On the basis of nucleotide sequence variations in the $N$ gene between nucleotides 1231 and 1686, a number of different lineages of MV have been identified, several of which can cocirculate in geographically restricted areas [13].

We compared the nucleotide sequence of the region corresponding to the $N$ region of $1 \mathrm{MV}$-BIL isolate by polymerase chain reaction amplification of $\mathrm{CDNA}$ obtained from MV-BILinfected B-LCL. On the basis of this analysis, MV-BIL appeared to be closely related to wild type MVs originating from lineages previously shown to circulate in Europe and the United States (data not shown). MV-BIL was subsequently cloned by a twofold limiting dilution of cell-free virus in B-LCL (B-LCL JP) [10]. Virus stocks of MV-BIL, containing $2 \times 10^{4} \mathrm{TCID}_{50} / \mathrm{mL}$, were prepared in the same cell line and kept at $-135^{\circ} \mathrm{C}$ until use.

Infection of cvnomolgus monkeys with different MV strains and reisolation of viruses. MV-seronegative cynomolgus monkeys (ages 20-30 months) were inoculated intratracheally (int) or intramuscularly $(\mathrm{im})$ with the following preparations: MV-BIL $\left(10^{-1.0}, 10,10^{1.0}, 10^{2.0}, 10^{3.0}\right.$, and $10^{4.0}$ TCID $_{50}$ int; 3 monkeys/ inoculum), the Edmonston strain of MV (MV-Edmonston; $10^{3.0}$ $\mathrm{TCID}_{50}$ int: 2 monkeys), and attenuated Schwartz measles vaccine (MV-Schwartz, Rouvax, lot G0334; I dose/animal int or im; 2 monkeys each; vaccine provided by Institut Mérieux, Lyon, France). MV-Edmonston had a history of six passages in human embryo kidney cells and three additional passages in Vero cells in the laboratory of P. Albrecht (Food and Drug Administration, Bethesda, MD), who donated this virus. All monkeys were monitored daily for development of clinical symptoms. At days 3, 5, 7, 9, 11, 14, and 18 after infection, nasopharyngeal washings and heparinized peripheral blood and lung lavages were collected for MV isolation by the following procedures.

PBMC were isolated from heparinized blood by centrifugation onto a gradient containing 4\% (wt/vol) dextran 500 (Sigma, St. Louis) and $32 \%(\mathrm{vol} / \mathrm{vol})$ of a standard metrizoate solution containing 32.8\% (wt/vol) natrium-metrizoate (Nycomed, Oslo). PBMC were cultured in 96-well round-bottomed plates ( 8 wells/sample) at a density of $8 \times 10^{4}$ cells/well in $150 \mu \mathrm{L}$ of RPMI 1640, supplemented with $10 \%$ ( $\mathrm{vol} / \mathrm{vol})$ fetal calf serum and antibiotics (FCS-medium) in the presence of $50 \mu \mathrm{g} / \mathrm{mL}$ phytohemagglutinin (PHA-M; Boehringer, Mannheim, Germany). After culture for $3 \mathrm{~h}$ at $37^{\circ} \mathrm{C}$, logarithmic dilutions of these cultures were cocultivated with B-LCL JP in round-bottomed wells at a density of $10^{4} \mathrm{~B}-\mathrm{LCL}$ per well. Lung lavages were centrifuged, and the cells were resuspended in FCS-medium. The cells were cultured in 24-well flat-bottomed microtiter plates (Greiner Labor Technik, Nürtingen, Germany) at a density of $2 \times 10^{5}$ cells/well ( 6 wells/sample) and cocultivated with $10^{5} \mathrm{~B}-\mathrm{LCL} \mathrm{JP} /$ well. Cells were collected from nasopharyngeal washings and cocultured with human B-LCL JP as described for lung lavage cells. All cultures were visually monitored for cytopathic changes over 10 days. The presence of MV-infected BLCL in cultures was demonstrated by immunofluorescence staining and by use of a $\mathrm{T}$ cell proliferation assay with MV-specific human $\mathrm{T}$ cell clones as previously described $[10,11]$.

Serologic assays. Plasma samples from heparinized blood were heat-inactivated for $30 \mathrm{~min}$ at $56^{\circ} \mathrm{C}$ and tested for the presence of MV-specific IgM and IgG antibodies in ELISAs and in virus-neutralizing assays. IgG-specific ELISAs and virus-neutralizing assays were done as described [12]. IgG titers were expressed as the reciprocals of dilutions of individual serum samples showing $50 \%$ of the maximal optical density value at 450 $\mathrm{nm}$ and virus-neutralizing antibody titers as the reciprocals of dilutions of sera showing 50\% inhibition of MV infection on Vero cells.

Specific IgM antibodies were detected by an antibody capture ELISA as described [10] with minor modifications. Briefly, microtiter plates (no. 3590; Costar, Cambridge, MA) were coated with 1:500 diluted $\mathrm{F}(\mathrm{ab})_{2}$ goat anti-human IgM antibodies (Organon Teknika-Cappel, West Chester, PA). Anti-IgM-coated plates were then incubated with 1:100 dilutions of individual monkey serum samples followed by an incubation with MV antigen $(20 \mu \mathrm{g} / \mathrm{mL}$ purified and $B$-propiolacton-inactivated $\mathrm{MV}$ ) [12]. Plates were subsequently incubated with 1:1000 diluted rabbit anti-MV antiserum [12] followed by an incubation with 1:500 diluted horseradish-peroxidase-conjugated swine antirabbit IgG (Amersham Laboratories, Amersham, UK). The latter two incubations were done in the presence of $2 \%(\mathrm{vol} / \mathrm{vol})$ normal monkey serum. Plates were developed and the absorbance was read at $450 \mathrm{~nm}$. IgM titers were expressed as optical density values.

\section{Results}

Virus isolation from MV-BIL-infected cynomolgus monkeys. Human and marmoset B lymphoblastoid cell lines have been shown to be sensitive for isolation and propagation of MV from clinical specimens of measles patients [ 10 , 14]. In a preliminary experiment, 1 isolate (MV-BIL) developed a rapid and extensive cell-associated viremia in cynomolgus monkeys after intravenous inoculation with cells infected with MV-BIL [10]. To study the susceptibility of cynomolgus monkeys to infection with cell-free MV-BIL via the respiratory route, $12 \mathrm{MV}$-seronegative animals were inoculated int with serial $10 \log$ dilutions of biologically cloned MV-BIL (10-10 $0^{4.0}$ TCID $_{50}$ ). Successful MV infection was demonstrated in all MV-BIL-infected animals by virus isolation through cocultivation of PBMC, lung lavage cells, and pharyngeal epithelial cells with human B-LCL JP (figure 1).

To obtain an indication of the minimal dose of MV-BIL infectious to cynomolgus monkeys, 2 additional animals were inoculated with 10 and $10^{-1.0} \mathrm{TCID}_{50} \mathrm{MV}$-BIL, respec- 
tively. The monkey inoculated with 10 TCID $_{50}$ MV-BIL became infected (figure 1). Animals infected with $10^{3.0}$ and $10^{4.0}$ TCID $_{50}$ MV-BIL had peaks of infectivity $\sim 7$ days after infection $\left(3-10 \times 10^{3.0} \mathrm{MV}\right.$-infected cells/ $10^{6} \mathrm{PBMC}$; figure 1). Although animals infected with lower infectious doses (10-10 $0^{1.0}$ TCID $\left._{50}\right)$ showed similar kinetics of PBMC-associated viremia, the peak of infectivity was delayed by 3-6 days. PBMC-associated viremia largely coincided with successful isolation of $\mathrm{MV}$ from lung lavage cells from an infected animal (figure 1). The presence of MV infection in pharyngeal epithelial cells could be demonstrated during and shortly after the peak of PBMC-associated viremia in most of the infected animals (figure 1).

Comparison of virus isolation and serum antibody response in MV-BIL-, MV-Edmonston-, and MV-Schwartz-infected cynomolgus monkeys. Two MV-seronegative monkeys were inoculated int with $10^{3.0}$ TCID $_{50} \mathrm{MV}$-Edmonston. Four animals were inoculated with $10^{3.0}$ TCID $_{50}$ MV-Schwartz, either int or im. Subsequently, reisolation of the viruses was done as described above. Isolates from animals infected with MV-Edmonston and attenuated MV-Schwartz were compared with those of $2 \mathrm{MV}$-BIL-infected animals. Monkeys infected with MV-Edmonston had kinetics of MV viremia similar to those of the MV-BIL-infected animals, but they had fewer MV-infected PBMC on day 7 after infection (10150/106 PBMC; figure 2). Because MV-Edmonston had a passage history on Vero cells, PBMC from MV-Edmonstoninfected monkeys were also cocultivated with Vero cells. Results were identical to cocultivation of PBMC with B-LCL (data not shown). Vaccination (im) of 2 monkeys with MVSchwartz resulted in low but detectable numbers of MV-infected PBMC in both monkeys (10-50/10 $0^{6}$ PBMC on days 9-11; figure 2). Infection (int) of 2 other monkeys with MVSchwartz resulted in low numbers of $M V$-infected PBMC at day 7 after infection in 1 of the 2 , but MV could be isolated from the lung lavage cells of both animals on days 5-7 after infection (data not shown). No pharyngeal epithelial cells could be isolated from MV-Edmonston- and MV-Schwartzinfected monkeys (data not shown).

Monkeys infected with MV-BIL, MV-Edmonston, and MV-Schwartz developed high, moderate, and moderate-tolow levels of specific serum IgM antibodies, respectively, which were detected over $\sim 3$ weeks, the onset of which coincided with the peaks of infectivity in individual animals (figure 2). The presence of MV-specific IgG serum antibodies, coinciding with the presence of MV neutralizing antibodies, was seen shortly after the appearance of specific IgM serum antibodies and gradually increased to plateau levels within $\sim 6$ weeks. MV neutralizing antibody titers of MVBIL-infected monkeys remained stable (512-2048) over the 9-month observation period (figure 2). One of the $2 \mathrm{MV}$-Edmonston-infected monkeys also developed a neutralizing

$\begin{array}{llllll}10,000 \mathrm{TCID}_{50} & { }^{1000 \mathrm{TCID}_{50}} & { }^{100 \mathrm{TCID}_{50}} & 10 \mathrm{TCID}_{50} & 1 \mathrm{TCID}_{50} & 0.1 \mathrm{TCID}_{50} \\ \text { monkeys 1,2,3 } & \text { monkeys 4,5,6 } & \text { monkeys } 7,8,9 & \text { monkeys } 10,11,12 & \text { monkey } 13 & \text { monkey } 14\end{array}$
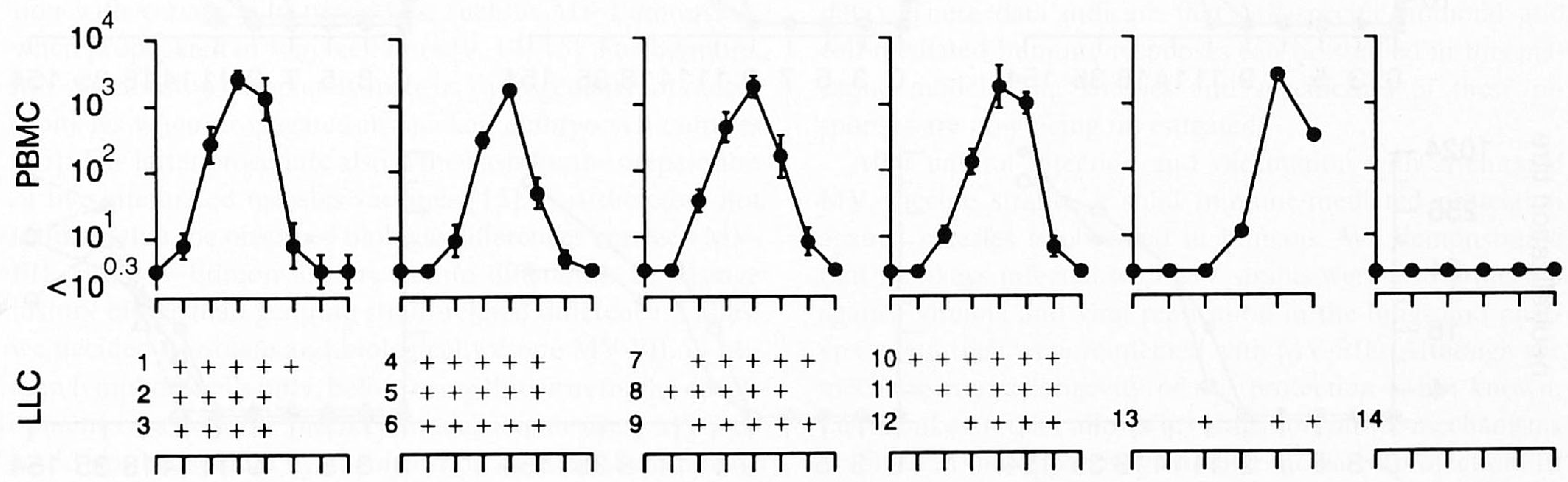

14
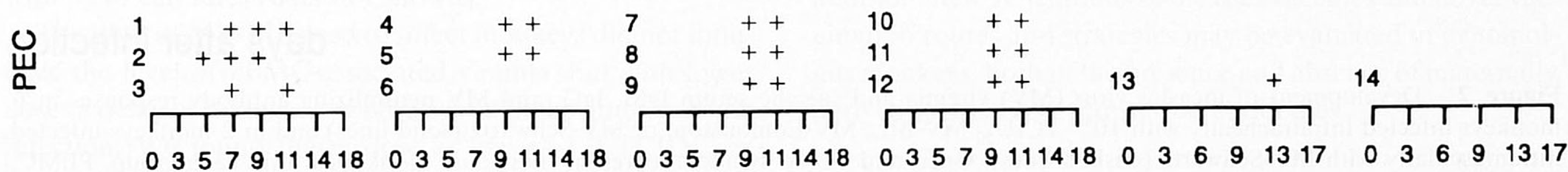

days after inoculation

Figure 1. Measles virus (MV)-infected cells in peripheral blood mononuclear cells (PBMC), lung lavage cells (LLC), and pharyngeal epithelial cells (PEC) of 14 cynomolgus monkeys infected intratracheally with different doses of wild type MV strain (MV-BIL). Graphs represent average numbers $( \pm \mathrm{SD})$ of $\mathrm{MV}$-infected cells $/ 10^{6} \mathrm{PBMC}$ per inoculum. + indicates successful MV isolations from LLC and PEC. 

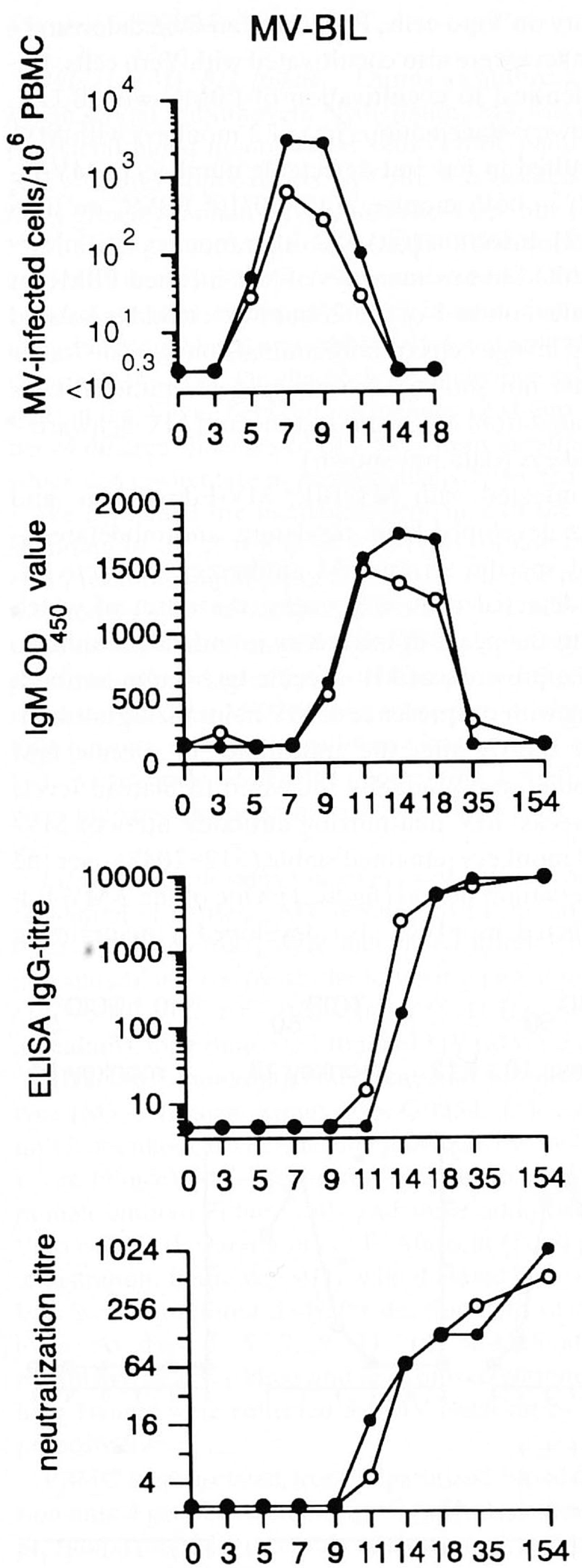

MV-Edmonston
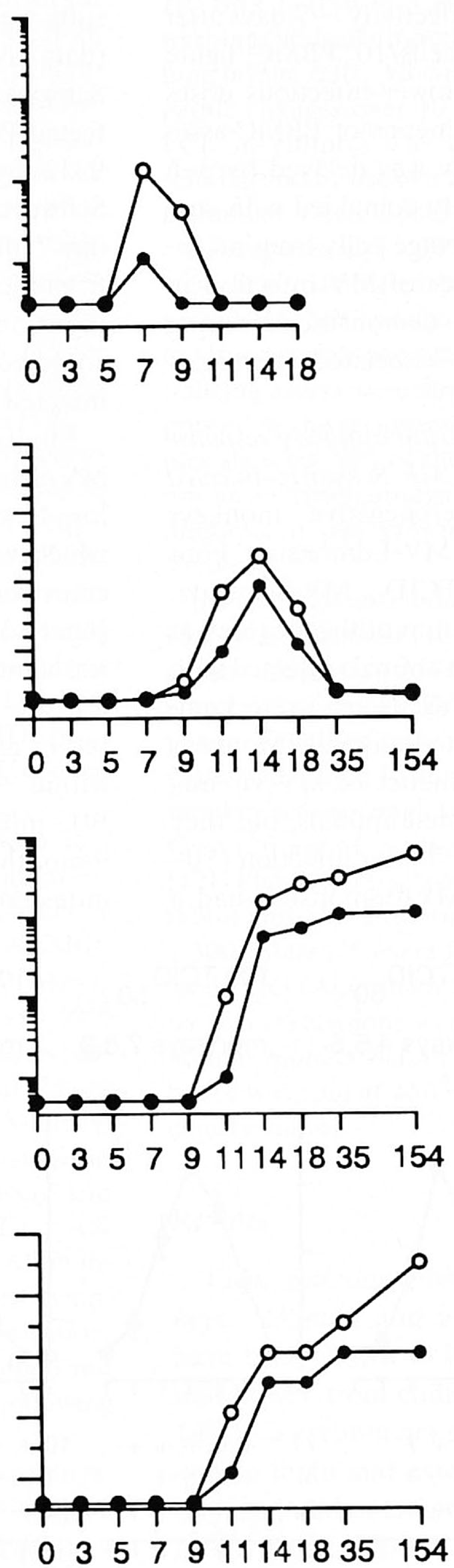

MV-Schwartz
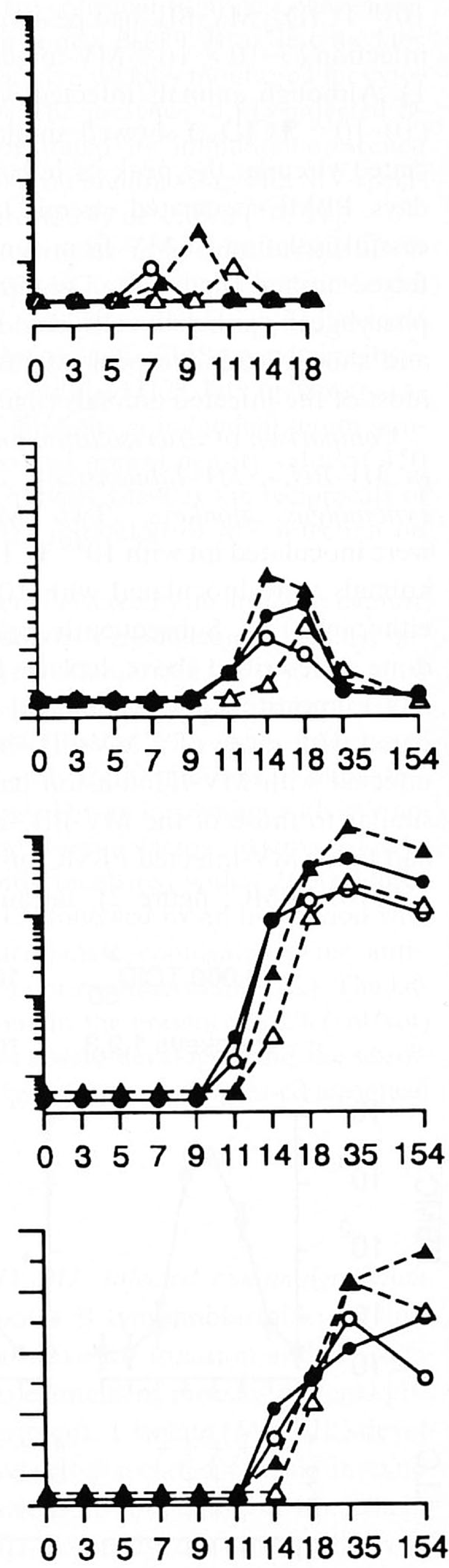

days after infection

Figure 2. Development of measles virus (MV) viremia and specific serum $\operatorname{IgM}$, IgG, and MV neutralizing antibody responses in 6 monkeys infected intratracheally with $10^{3.0} \mathrm{TCID}_{50} \mathrm{MV}$-BIL. MV-Edmonston, or MV-Schwartz (solid lines) and in 2 monkeys infected intramuscularly with MV-Schwartz (dashed lines). Open and closed symbols represent individual monkeys within each group. PBMC, peripheral blood mononuclear cells. 
antibody titer of 1024, whereas the other and all MVSchwartz-infected monkeys developed lower MV neutralizing antibody titers (32-256).

Protection from reinfection with MV-BIL after infection with different MV strains. Two MV-seronegative control animals and all of the MV-infected monkeys shown in figure 2 were challenged int with $10^{3.0}$ TCID $_{50}$ MV-BIL 9 months after initial MV infection. They were monitored for development of PBMC-associated viremia, infected cells in lung lavages and pharyngeal washings, and changes in MV-specific IgM and IgG serum antibody titers. No MV-infected cells were seen in any of the monkeys in figure 2 in the 3 weeks after challenge. Accordingly, none of these animals showed specific serum antibody titer rises. The 2 controls included in this challenge experiment had patterns of $\mathrm{MV}$ isolation and serology similar to the other monkeys infected int with $10^{3.0}$ TCID $_{50}$ MV-BIL (not shown).

\section{Discussion}

Our results show that cynomolgus monkeys can be infected with MV-BIL, a recently isolated strain that belongs to a cluster of wild type MVs now circulating in the United States and Europe [10, 13]. Monkeys infected with MV-BIL developed higher levels of PBMC-associated viremia than did monkeys infected with MV-Edmonston; MV-infected cells were also found in lung lavages and pharyngeal swabs at different times after infection.

Previous studies have shown that rhesus and cynomolgus monkeys have limited susceptibility to experimental infection with certain wild type MVs, such as MV-Edmonston, when propagated in Vero cell lines [9, 14, 15]. Furthermore, MV-Edmonston completely loses its pathogenicity for rhesus monkeys when propagated in chicken embryo cell cultures [15]. The latter procedure also is the basis for the preparation of live attenuated measles vaccines [15]. It is therefore not unlikely that the observed biologic differences between MVBIL and MV-Edmonston are due to differences in passage history rather than genuine strain-related differences. Thus, we decided to isolate and biologically clone MV-BIL in human lymphoid cells only, before using this virus for the development of a suitable monkey model for measles. MV-BIL could not be isolated by cocultivation of clinical specimens with Vero cell lines (data not shown).

The dose of MV-BIL used to infect monkeys did not influence the level of PBMC-associated viremia, but with lower doses a delay of some days was observed in the kinetics of the infection. We found that a dose as low as $1.0 \mathrm{TCID}_{50}$, as measured in human B-LCL, corresponded with $\sim 1$ monkey infectious dose (figure 1). This confirms the high infectious nature of $\mathrm{MV}$, which has also been seen in humans under natural conditions $[1,7]$. Infection of monkeys via different routes (int or im) with MV-Schwartz, an attenuated vaccine strain, resulted in lower levels of viremia than observed with
MV-BIL and MV-Edmonston (figure 2). The im route was included in these experiments to mimic the normal route of vaccination [7].

It may be speculated that the levels of PBMC-associated viremia and the presence or absence of $\mathrm{MV}$-infected cells in lung lavages and in pharyngeal washings measured in macaques are parameters of MV virulence. In this respect, even a low passage of MV-Edmonston could be regarded as indicating an attenuated MV strain that would not be predicted on basis of its biologic properties in vitro (our findings and [14]). However, as none of the infected animals developed major clinical symptoms (only MV-BIL-infected monkeys showed a transient and mild lethargy and temperature rise; data not shown), we cannot yet conclude whether the biologic properties of the different MV strains will correlate in humans and macaques.

Comparison of serum antibody responses following infection with MV-BIL, MV-Edmonston, or MV-Schwartz showed that the $\operatorname{IgM}$ responses had the same trends as seen in PBMC-associated viremia. These differences were less pronounced for MV-specific serum IgG levels and MV neutralizing antibodies (figure 2). MV neutralizing antibody titers of MV-BIL-infected monkeys were similar to those seen in sera from children after infection with MV-BIL (figure 2; data not shown). MV-BIL infection in the children also coincided with strong $\mathrm{MV}$-specific proliferative $\mathrm{CD} 4^{+}$and $\mathrm{CD}^{+} \mathrm{T}$ cell responses in their PBMC $[2,10,11]$. By using similar techniques, we demonstrated the expansion of specific $\mathrm{CD}^{+}$and $\mathrm{CD}^{+} \mathrm{T}$ cell populations in the PBMC of monkeys previously infected with MV-BIL and MV-Schwartz (unpublished data). These data indicate that MV-specific humoral and cell-mediated immune responses can be studied in this macaque model. The kinetics and specificities of these responses are now being investigated.

After natural infection and vaccination with attenuated MV vaccine strains, a solid immune-mediated protection against measles is observed in humans. We demonstrated that monkeys infected with MV strains were also protected against viremia and viral replication in the lungs and phar$y n x$ when they were reinfected with MV-BIL. Although the mechanism and longevity of this protection is not known, this monkey model allows investigation of the mechanisms involved in this apparently immune-mediated protection. In addition, new generations of measles vaccines and novel vaccination routes and strategies may be evaluated in cynomolgus monkeys, both in the presence and absence of maternally derived or passively transferred antibodies.

\section{Acknowledgments}

We thank Bert Rima, Nico Schmidt, Jan Groen, Martien Poelen, and Carmen Jansen for technical assistance and Conny Kruyssen for help in manuscript preparation. 


\section{References}

1. Norrby E. Measles vaccination, today and tomorrow. Ann Inst Pasteur/ Virol 1985:136E:56I-70.

2. UytdeHaag FGCM, van Binnendijk RS, Kenter MJH, Osterhaus ADME. Cytotoxic T lymphocyte responses against measles virus. Curr Top Microbiol Immunol 1994:189:151-67.

3. Gustafson TL, Lievens AW, Brunnel PA, Moellenberg RG, Buttery CMG. Sehulster LM. Measles outbreak in a fully immunized secondary-school population. N Engl J Med 1987:316:771-4.

4. Chen RT. Markowitz LE. Albrecht P. et al. Measles antibody: reevaluation of protective titers. J Infect Dis 1990;162:1036-42.

5. Markowitz LE. Preblud SR, Orenstein WA. et al. Patterns of transmission in measles outbreaks in the United States, 1985-1986. N Engl J Med 1989:320:75-81.

6. Weiss R. Measles battle loses potent weapon. Science 1992;258:546-7.

7. Expanded Programme on Immunization. Measles control in the 1990s: plan of action for global measles control. Geneva: World Health Organization, 1992:WHO/EPI/Gen/92.3.

8. van Binnendijk RS, van der Heijden RWJ. Osterhaus ADME. Monkeys in measles research. Curr Top Microbiol Immunol 1994 (in press).

9. Albrecht P. Lorenz D. Klutch MJ, Vicjers JH. Ennis FA. Fatal measles infection in marmoset pathogenesis and prophylaxis. Infect Immun 1980;27:969-78.
10. van Binnendijk RS, Rümke HC, Van Eijndhoven MJ, et al. A measles outbreak in vaccinated schoolaged children in the Netherlands: identification of clinically and subclinically infected children by evaluation of virus-specific antibody and $T$ cell responses. In: T cell function in measles [doctoral thesis]. Chapter 2. Utrecht, Netherlands: University of Utrecht. 1992.

11. van Binnendijk RS, Versteeg-van Oosten JPM, Poelen MCM, et al. Human HLA class I- and II-restricted cloned cytotoxic T lymphocytes identify a cluster of epitopes on the measles virus fusion protein. J Virol 1993;67:2276-84.

12. De Vries P. van Binnendijk RS, van der Marel P, et al. Measles virus fusion protein presented in immune-stimulating complex (ISCOM) induces haemolysis-inhibiting and fusion-inhibiting antibodies, virus-specific $T$ cells and protection in mice. $J$ Gen Virol 1988:69:549-59

13. Taylor MJ. Godfrey E. Baczko K, ter Meulen V. Wild TF, Rima BK. Identification of several lineages of measles virus. J Gen Virol 1992:72:83-8.

14. Kobune F. Skata H. Sugiura A. Marmoset lymphoblastoid cells as a sensitive host for isolation of measles virus. J Virol 1990;64:700-5.

15. Enders JF. Katz SL. Milovanovic MV. Holloway A. Studies on an attenuated measles-virus vaccine. I. Development and preparation of the vaccine: techniques for assay of effects of vaccination. N Engl J Med 1960:263:153-9. 